\title{
Selective polarization of ferroelectrics in functional electronics
}

\author{
Ya.I. Lepikh \\ Scientific-Technologiccal Center «Phonon», SCTB «Element» \\ 29, pr. Acad. Glushko, 270104 Odessa, Ukraine, Tel. (0484)-66-82-29, Fax.: (0482)-47-02-23
}

\begin{abstract}
The selective polarization technique of ferroelectrics representing sound-conductors and piezoelectric transformers in different devices of functional electronics is described. The feature of the technique is the ability of creating the local polarization regions, with different, in the magnitude and orientation, polarization vectors and with different electrophysical parameters, in the monolithic ceramic sound-conductor. The examples of specific acousto-electron units based on surface acoustic waves with the essentially improved electrical parameters, simpler construction and higher processibility are presented. The features of the selective polarization process are shown, the recommendations on the usage of the technique are given.
\end{abstract}

Keywords: ferroelectric, domain, sound conductor, polarization, converter, wave, parameter.

Paper received 27.05.99; revised manuscript received 20.09.99; accepted for publication 15.10.99.

One of directions for the efficient improving the characteristics of functional electronic (FE) (acousto-, optoelectronics, acousto-optics, etc.) devices is the development of new functional materials with controllable properties and improvement of techniques for the maximum realization of their potentials. The perspectives of such a direction of studies are more promising than the design improvement. This is confirmed by the achievement of high values of specific parameters of FE devices due to application of ferroelectrics, $\mathrm{A}^{\mathrm{II}} \mathrm{B}^{\mathrm{VI}}$ structures and other materials [1-4].

The acoustoelectronic devices on surface acoustic waves (SAW), for example, having evident advantages as compared to devices based on other physical principles, sometimes are inferior due to relatively large magnitude of inherent signal losses. This problem is often solved using complicated design of interdigital transformers (IDT) or by introduction of supplementary radio devices $[4,5]$. In the first case, the one-directional IDT, multiple-strip couplers, in the second case, the phase-shifters are used. But in both cases such solutions make the device construction much more complex, reduce their processibility and often are not efficient.

The attempts were made [6] to reduce the induced losses by variation of electrophysical parameters (EPP) of the sound-conductor piezoelectric material using the additional polarization of piezoceramics. In this case the complex procedure of power forming signal generation with a complex acoustic wave spectrum of a given form.

We suggest a technique for improving the SAW-based device characteristics by application of selective polariza- tion of ferroelectrics used as sound-conductors and piezoelectric transformers. This technique may be also used for another kinds of FE devices. In the SAW-based soundconductor containing the IDT system, the regions are formed with polarization vectors $\overrightarrow{\mathrm{P}}$ with different both amplitude and orientation in respect to the operation surface of the sound-conductor (Fig.1). In this case, in the region where the input IDT is situated the polarization vector $\overrightarrow{\mathrm{P}}_{1}$ is directed normally to the sound-conductor operation surface, and in the region where the output IDT is situated, the polarization vector $\overrightarrow{\mathrm{P}}_{2}$ is parallel to it, so that $\left|\mathrm{P}_{2}\right|>\left|\mathrm{P}_{1}\right|$. The part of the sound-conductor between IDT can be non-polarized, i.e. $\overrightarrow{\mathrm{P}}_{3}=0$.

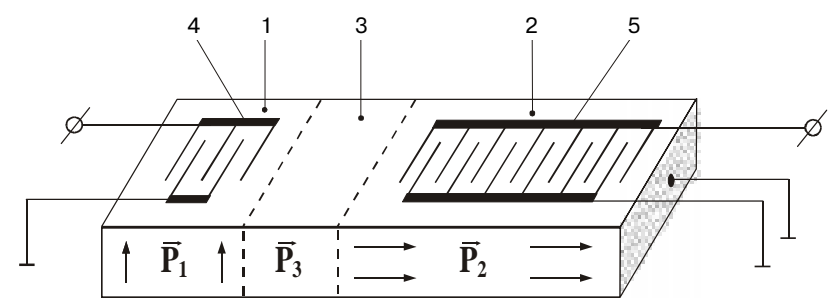

Fig.1. The block diagram of SAW device with selectively polarized piesoceramic sound-conductor. 1 - region with transverse polarization, 2 - region with longitudinal polarization, 3 - non-polarized region, 4 - input IDT, 5 - output IDT. 


\section{Ya. I. Lepikh: Selective polarization of ferroelectrics in functional electronics.}

Thus, in the parts of the piezoceramic sound-conductor with longitudinal-transverse polarization the regions are formed with different values of EPP, in particular, of electromechanical coupling factor $K_{P}$ and dielectric constant $\varepsilon$. The onset of input and output IDT of the SAW device is performed in such way that one of summation buses of IDT and conductive layers situated on inactive surface of the sound-conductor, are grounded like as in the piezoceramic transformer. Due to a higher value of $K_{P}$ in the region $\overrightarrow{\mathrm{P}}_{2}$ and due to a greater area from which the signal is collected by more extended output IDT, the voltage transformation of the electrical signal generated by the output IDT occurs.

Using this technique the samples of the SAW bandwidth filters with the mean frequency $f_{0}=10 \mathrm{MHz}$ were fabricated. The piezoceramics of plumbum zirconat-titanat PZT42 was used. In the experimental filter samples the magnitude of induced signal losses was less than $2 \mathrm{~dB}$, which can not be achieved by other techniques.

The possibilities of ferroelectric selective polarization are not restricted to creation of local parts with different polarization in the monolithic sound-conductor. By formation of the polarization regions of different geometrical shape it is possible, in particular, to form the amplitudefrequency characteristics of SAW devices. In [7], for example, to reduce the distortions of the SAW phase front the weighing of IDT electrodes with the same overlapping was performed by setting the polarized region with a definite shape in the IDT plane. In particular, for the bandwidth filter the configuration of the region (Fig.2) had the shape of the envelope of its pulse characteristic $h(t)$ obtained from its given transfer characteristic $H(\omega)$ by the reverse Fourier transform $h(t)=\int_{-\infty}^{\infty} H_{o}(\omega) \exp (i \omega t) \mathrm{d} \omega$, where $\omega$ is the circular frequency. At the same time, such technique of IDT apodization allows to increase essentially its processibility, since it removes the strict requirement imposed on the fabrication precision of small IDT electrode bridges, corresponding to calculated weighting functions.

Even greater successes of application of the selective polarization are expected in acousto-optics, optoelectronics, where the ferroelectric ceramics based on lanthanum

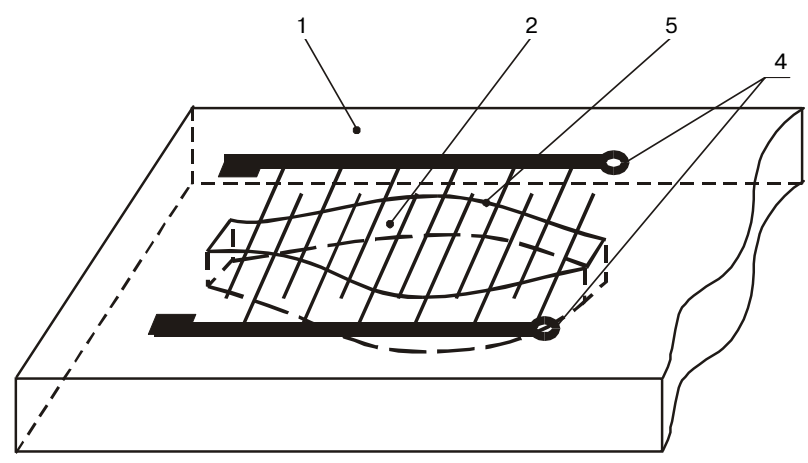

Fig.2. The diagram of IDT apodization by polarization with a given shape of polarized region. 1- sound conductor, 2- polarized region of ferroceramics, 3- envelope of apodization function, 4- IDT. doped PZT (PLZT) with high optical transparency, sensitivity, stability and other advantages $[8,9]$ is applied more and more intensively. It is supposed that the method will enhance also the sensor technology potential.

In this technique all procedures of piezoceramic substrate fabrication from the ferroelectric material (the synthesis of compound, formation, sintering, mechanical treatment) prior to polarization do not differ from the conventional ones. While using the selective polarization, which can be carried out using the set-up described in [10] this process is slightly different.

Omitting the quantum-mechanical aspect of the atom behaviour in the ferroceramic crystal structure, one should take into account, that at longitudinal polarization, when the external electric field $\overrightarrow{\mathrm{E}}$ is directed normally to the spontaneous polarization vector $\overrightarrow{\mathrm{P}}_{\mathrm{s}}$, i.e. along one of directions [100] or [010], the dipole polarization mechanism is prevalent with a 90-degree turn of domains. Since the coercive field of 90-degree domain turn is higher (the cell deformation occurs), than that of 180-degree turn, the longitudinal polarization mode should differ by the polarization field $\overrightarrow{\mathrm{E}}$ magnitude, temperature $\mathrm{K}$ and polarization time $t_{p}$. At 90degree domain reorientation strong mechanical stresses appear, which, together with the field accumulated in the nearelectrode space-charge regions, after completing the polarization lead to a greater change of piezoceramic parameters, than in the case of 180-degree reorientation. It should be noted that due to different coercive fields of ferroelectrics with different composition, at the dipole type of polarization under the external field the qualitatively different polarization results are reached. For example, in PZT ceramics the number of domains that are 90-degree reorientable is by a factor of 3-3.5 greater than in barium titanat $\left(\mathrm{BaTiO}_{3}\right)$. These features are manifested in the process of the piezoceramics aging. This should be taken into account during a device calculation, using the stabilized EPP values, which are reached for the longer time interval, or by artificial acceleration of aging.

The technique of ferroelectric selective polarization can be used for the solution of a broad range of problems in acousto- and optoelectronics, acousto-optics and other directions of functional and solid-state microelectronics.

\section{References}

1. New materials for radio-, opto- and acoustoelectronics. Inter-university proceedings. Krasnoyarsk: Izd. KGU, 1982.-158 p.

2. Yu.V. Guliaev, Ya.I. Lepikh. SAW intermediate frequency filter with piezo-ceramic sound-conductor. In: X All-union acoustic conference. Talks. Section V.M.: 1983, p.16-19.

3. Ya.I. Lepikh. Application of PZT-based ceramics in SAW devices. Elektronnaya tekhnika. Ser. 10. Mikroelektronnye ustroistva. 1988, ser. 5, p.48-50.

4. F.G. Marshall, C.O. Newton, E.G.S. Paige. Surface acoustic wave multistrip components and their applications. IEEE Trans., vol. MTT21, 1973, pp. 216-224.

5. K. Yamanouchi, T. Meguro and K. Qautam. Low-loss GHz range SAW filter using group-type unidirectional transducer-new GUDT and new phase shifter. IEEE Ultrasonics Sump., 1982, pp. 212-217.

6. A.c. USSR No.614530, NOZN 9/30 publ. 5.07.78 in bul. No.25. 
Ya. I. Lepikh: Selective polarization of ferroelectrics in functional electronics.

7. Ya.I. Lepikh. Formation of the characteristics of SAW devices using the selective polarization. In: Proceedings of International Scie.-Pract. Conference «Basic problems of modern piezoelectronics» Rostovon-Don. MP «Kniga». 1995, v.3, p.152-153.

8. A.R. Shternberg. Electrooptical ferroelectricity.- Riga, LGU, 1975 v.230, p.21-133
9. V.I. Balakshiy, V.N. Parygin, L.E. Chirkov. Physical bases of acoustooptics.- M.: Radio i Sviaz', 1985.- 279 p.

10. Ya.I. Lepikh. Automated set-up for ferroceramic polarization. Pribory i Tekhnika Eksperimenta.- 1996, No.4, p.146. 